\title{
Stimulus control: Contrast, peak shift, and Konorskian models
}

\author{
G. BIGNAMI, DEPARTMENT OF THERAPEUTIC CHEMISTRY, \\ ISTITUTO SUPERIORE di SANITA, Roma, Italy
}

The controversy on inhibition in operant discriminations is examined in the light of Konorski's models. Errorless learning apparently leads to "pure" drive inhibition by S-s. Nonreception of anticipated rewards during learning with errors can provoke both drive enhancement, and drive and response inhibition by $S$-s sharing some of the excitatory properties of $S+s$. These differences, rather than inhibitory phenomena per se, can explain contrast, peak shift, and U-shaped gradients of inhibition after learning with errors.

Terrace $(1963,1966 \mathrm{a}, 1966 \mathrm{~b}, 1967)$ has shown that successive discriminations learned without errors, i.e., without periods of substantial responding during the presentation of the negative stimulus (S-), differ in several regards from discriminations learned with errors. Behavioral contrast (increased responding to the positive stimulus, $S+$, after the introduction of $S-$ ), peak shift (displacement of the peak of the generalization gradient around $\mathrm{S}+$ in a direction opposite to that of S-), and U-shaped gradients of inhibition around S- are observed only if the animals make several errors of commission during training. Terrace has interpreted these results as showing that only learning with errors leads to a dual stimulus control of discrimination, namely excitatory control by $\mathrm{S}+$ and inhibitory control by $\mathrm{S}$-. This hypothesis has been questioned by Deutsch (1967), who argued that a flat gradient around $S$ - after errorless learning, obtained with response rates close to zero, lends itself to several interpretations, including that of a stronger inhibition by S-. Furthermore, Biederman (1967) has shown that a neutral stimulus is preferred to $S$ - after learning a simultaneous discrimination with or without errors.

The purpose of the present note is to examine this controversy on the relative role of $S+$ and $S$ - in the light of some of the models proposed by Konorski (1967) to explain a vast body of data on classicaland instrumental conditioning. Two points must be given special consideration, namely the hypothesis of dual driveantidrive systems, and the analysis of the differences between an ordinary S- and a "Primary Inhibitory Stimulus" (PS-).

Drive and Antidrive. The old concepts of "drive excitation" and "drive inhibition" acquire an entirely new meaning in the revised Konorskian models. This makes necessary a brief description of the proposed drive-antidrive system, so as to avoid a gross distortion of Konorski's hypotheses. The previous models, based on an algebraic summation of excitatory and inhibitory influences on drive, do not account for various experimental data, e.g., the lack of symmetry between extinction curves and restoration curves after the reintroduction of reinforcement. Konorski suggests that this and other phenomena could be explained by postulating that functional connections are formed between stimuli and drive (formerly excitatory connections) ${ }^{1}$ and between stimuli and antidrive (formerly inhibitory connections), so as to leave to the antidrive system the role of suppressing the activity of the drive system. In other words, the hypothesis uses only one basic type of change provoked by experience, and two types of mutually antagonistic systems established in ontogeny. Admittedly, the neurophysiological evidence on built-in antagonistic mechanisms responsible for hunger, thirst, fear, etc. has played a role in the construction of the new model.

PS- and S-. Several sections of Konorski's monograph are devoted to classical differentiation learned with and without errors, i.e., with and without initial responding to S-. Konorski and his associates ${ }^{2}$ have analyzed in detail the properties of PS-, i.e., a nonreinforced stimulus presented among other reinforced stimuli, but situated outside the generalization range of the latter. Inhibitory properties can be shown by pairing the PS- with an S+, and observing a diminished response to $\mathrm{S}+$; and by using the PS- as
S+ for further conditioning tests, and observing that it is much less effective than other stimuli. A PS- and an ordinary S-, i.e., a negative stimulus originally situated within the range of generalization of $\mathbf{S}+$, differ in many respects. By definition, an S- eliciting response must share some of the excitatory properties of S+, progressively counteracted by the repeated association with nonreinforcement which leads to drive inhibition. A PS- does not possess excitatory properties, and can only acquire uncontaminated inhibitory properties. Furthermore, only an S- eliciting response, but not a PS-, can provoke the well-documented drive enhancement which follows the omission of a positive reinforcement at a time when it is anticipated. As one moves from the level of classical conditioning to that of instrumental conditioning another difference clearly appears. In fact, only an S- eliciting nonreinforced responses can induce the retroactive inhibition of these responses, which do not lead to drive reduction.

Konorski also gives evidence in favor of a direct motor act inhibition by S- (not mediated by drive inhibition). Further comments on this phenomenon, however, cannot be made, as Konorski agrees that a satisfactory block model cannot be designed on the basis of available evidence.

Stimulus Control in Discriminations. The models discussed in the two previous sections can be fitted in with the data mentioned in the first section with so little difficulty, that one could infer that these data represent an independent confirmation of the models themselves. Contrast after learning with errors can be explained by the fact that only an S- eliciting responses triggers the drive enhancement due to the omission of an anticipated reinforcement. Response rates during $\mathrm{S}+$ must return to normal when $S$ - becomes a clear signal of nonreinforcement, since anticipation of nonreinforcement and nonreception of an anticipated reinforcement have opposite effects on drive. An Soriginally not responded to must give a flat gradient of inhibition with response rates close to zero, assuming that stimuli similar to it also lie outside the original range of generalization of $\mathrm{S}+$. U-shaped gradients around S- after learning with errors can be explained by assuming that not only the S-, but also the stimuli similar to it, share some of the excitatory properties of S+, while specific no-go training confers maximal inhibitory properties to S- ${ }^{3}$ The occurrence of a peak shift after learning with errors can be explained by the fact that retroactive response inhibition and direct motor act inhibition can take place only if the S- is responded to. If specific retroactive inhibition and/or specific motor act inhibition are the principal factors, peak shifts should not be observed when measuring the generalization gradient with a response different from that used for discrimination training. Should a peak shift be observed also with a different response, this could be ascribed either to a much greater drive inhibition by $S$ after learning with errors, or to a generalized retroactive response inhibition. In fact, it is conceivable that exposure to a stimulus situation in which reinforcement is expected, and no type of response can procure it, may lead to a retroactive inhibition involving large sections of the behavioral repertoire. This phenomenon would still be compatible with the Konorskian model of retroactive response inhibition, extended to the whole range of motor behaviors occurring during prolonged presentations of S-.

Conclusion. Several points cannot be dealt with in detail in the present note. For example, the partial reinforcement schedule during $S+$ presentation in the experiments of Terrace may require a complication, but not a major modification, of the models. An extension of the experiment of Biederman (1967) to different conditions could be a source of information on the relative role of drive inhibition, of retroactive response inhibition mediated by drive inhibition, and of direct motor act inhibition. After discrimination training, one should perform preference studies with the $S$ - and a neutral stimulus in situations not associated with 
reinforcement, or using satiated animals. In case of a disappearance of the preference with satiation, the role of drive inhibition by S- could be confirmed by a reappearance of the preference with electrical stimulation of the hunger center, which is known to restore instrumental movements in satiated animals. Finally, the experiments of Terrace and those of the Konorski group provide important evidence on the role of stimulus generalization. It clearly appears that, when an organism is confronted with a novel stimulus in a situation in which other stimuli have already acquired a biological significance, the course of events depends entirely on whether or not the novel stimulus is interpreted as having the same biological significance as the others. Given a certain difference between two stimuli, both organismic and training factors influence the outcome. The former are demonstrated by experiments in which only one training procedure is used, and the animals are classified a posteriori on the basis of response or absence of response to $\mathrm{S}$ - (e.g., Terrace, 1966b; Biederman, 1967). The latter are shown by experiments in which particular shaping procedures are used to minimize the probability of responses to S- (e.g., Terrace, 1963). By extending these considerations to behavior in natural habitats, one could account for a tremendous amount of individual variability in response to identical environmental conditions.

\section{REFERENCES}

BIEDERMAN, G. B. Stimulus control in errorless discrimination. Psychon. Sci. 1967, 9, 375-376.
DEUTSCH, J. A. Discrimination learning and inhibition. Science, 1967, 156, 988.

KONORSKI, J. Integrative activity of the brain. Chicago: The University of Chicago Press, 1967.

TERRACE, H. S. Discrimination learning with and without "errors." J. exp. Anal. Behav., 1963, 6, 1-27.

TERRACE, H. S. Stimulus control. In W. K. Honig (Ed.), Operant behavior: areas of research and application. New York: Appleton-Century-Crofts, 1966 .

TERRACE, H. S. Discrimination learning and inhibition. Science, 1966b, $154,1677-1680$.

TERRACE, H. S. Discrimination learning and inhibition. Science, 1967, 156, 988-989.

\section{NOTES}

1. Imprecise expressions such as "functional connections between stimuli and drives, etc." are used as abbreviations of more cumbersome expressions such as "functional connections between neurophysiological systems activated by stimuli and neurophysiological systems subserving drive, etc.."

2. For reasons of space, the work of Konorski and his associates of the Nencki Institute in Warsaw is quoted by referring to the recent monograph (Konorski, 1967). The author wishes to apologize for this necessary omission of the names of many distinguished investigators.

3. Obviously, the symmetry or asymmetry of the slopes on either side of Smust depend on several factors (e.g., St and S- selected from the same or different continua). 